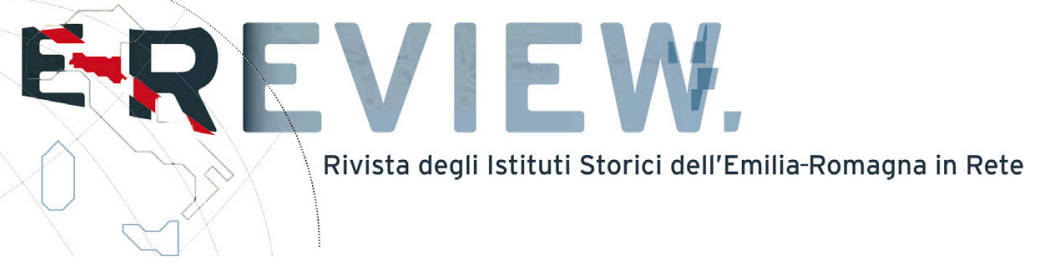

$\frac{\text { 8-9, 2021-2022 }}{\text { \#dossier }}$ 


\section{E-REVIEW}

Rivista degli Istituti Storici dell'Emilia-Romagna in Rete

Iara Meloni

Scolpiti nella memoria. Statue, commemorazioni e luoghi

di memoria della Resistenza a Piacenza

(c) Iara Meloni

Creative Commons BY-NC-ND 4.0

International License 2004-2021

Volume: 2021-2022

Issue: 8-9

Section: \#dossier - sul piedistallo della storia

Pages: 1-29

DOI: $10.52056 / 9788833138756 / 02$

ISSN: $1825-411 \mathrm{X}$

Publisher: Viella

Double blind peer review: Yes

Document type: Article

Research Areas: History

Published: December 2021

Corresponding Address: Iara Meloni, Università di Milano, Dipartimento di Studi storici, via Festa del Perdono 7 - 20122 Milano, Italy - Museo della Resistenza piacentina, strada provinciale 21 4, 29020 Sperongia di Morfasso (Piacenza), Italy 


\title{
Scolpiti nella memoria Statue, commemorazioni e luoghi di memoria della Resistenza a Piacenza
}

\author{
IARA MELONI \\ Università di Milano - Museo della Resistenza piacentina \\ iara.meloni@unimi.it
}

\begin{abstract}
Il saggio intende indagare le forme della costruzione della memoria della Resistenza dal dopoguerra a oggi, a livello nazionale $e$ in chiave locale, con un focus sulla provincia di Piacenza, al confine tra Emilia-Romagna e Lombardia e teatro di alcuni importanti conflitti interni al movimento resistenziale. Una particolare attenzione ̀̀ stata rivolta alle statue di partigiani realizzate dallo scultore Secondo Tizzoni, e a come le pratiche commemorative e le ritualità civili legate alla Resistenza si siano modificate negli anni.

Parole chiave: Piacenza, statue, Secondo Tizzoni,

EMILIO CANZI, MEMORIA DELLA RESISTENZA
\end{abstract}

Engraved in Memory. Statues, Commemorations and Sites of Memory of the Resistance in Piacenza.

The essay aims to investigate the remembrance of the Resistance since the postwar period to nowadays, with a focus on the province of Piacenza, at the border between EmiliaRomagna and Lombardia, which was the scene of some emblematic conflicts within the resistance movement. Through the decades, the commemorative practice have changed, and statues representing partisan martyrs have transformed their role.

Keywords: Piacenza, statues, Secondo Tizzoni, EMILIO CANZI, RESISTANCE MEMORY

\section{Introduzione}

Tramandano che lo scultore di ben conoscere quelle passioni rivelava Che ancor sopravvivono, stampate senza vita su queste pietre, Alla mano che le plasmava, e al sentimento che le alimentava.

Percy Bysshe Shelley, Ozymandias, 1818

Questo saggio intende riflettere sulle forme della costruzione della memoria resistenziale in provincia di Piacenza, mettendo al centro il tema delle statue e della monumentalizzazione della Resistenza. Di questo percorso si intende dare un quadro di longue durée, che va dalle prime manifestazioni post-belliche dedicate ai caduti alle forme attuali della commemorazione di un evento storico sentito come pregnante e fondativo. Si intende così collocare la storia locale, 


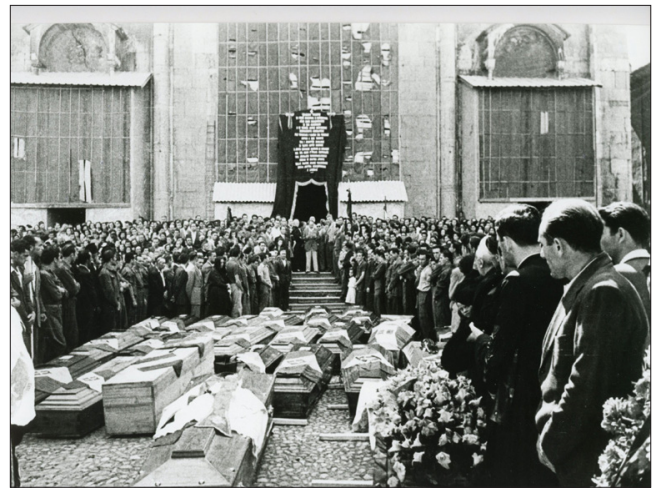

Fig. 1. Piacenza, luglio 1945. Funerale collettivo di partigiani caduti [Archivio Gruppo ricerca immagine, Bettola].

con le sue peculiarità e le sue piccole storie, nel più ampio quadro di un filone storiografico ormai solido e ben percorso.

I modi in cui la Resistenza è stata raccontata (nei contesti famigliari, comunitari e sulla scena pubblica) sono stati infatti a partire dagli anni Ottanta al centro della riflessione storiografica. Si è così proficuamente riflettuto sulle dinamiche e i vettori di costruzione della memoria della lotta di liberazione, concentrando l'attenzione inizialmente sulla letteratura [Falaschi 1976; Bianchini, Lolli 1997] e sulle arti figurative, e più avanti sul discorso pubblico, sulla memoria istituzionale, sul calendario civile. Gli storici si sono concentrati sulla comunicazione politica, sui testi giornalistici [Focardi 2005; Focardi 2020], sulle culture popolari [Winterhalter 2010; Cooke 2015], sul cinema [Cigognetti, Servetti, Sorlin 1996; Forgacs 1999], sulla televisione [Crainz 2000], sulla musica [Pivato 2005], sulla rappresentazione fotografica [Mignemi 1995; Giovannini Luca, Tabor 2017], sulle celebrazioni del 25 aprile [Paggi 1999; Ridolfi 2003]. Anche alcuni controversi episodi di strage hanno spinto gli storici a misurarsi con il difficile compito di decostruire memorie pubbliche e comunitarie, rivelatesi fallaci, stratificate, problematiche [Contini 1997; Portelli 1999; Baldissara, Pezzino 2009].

In questo quadro complessivo, al tema dei monumenti alla Resistenza - con alcune eccezioni ${ }^{1}$ - non è stata dedicata molta attenzione.

Questo silenzio risalta ancora di più tenendo conto della proliferazione di monumenti alla Prima guerra mondiale, che gli storici dell'arte hanno letto in continuità con la produzione monumentale ottocentesca, celebrativa del mito e dell'unità nazionale. A partire dai lavori di Mosse [Mosse 1990] lo studio delle forme della costruzione del mito dei caduti e di una nuova religione della patria dopo la Grande Guerra è stato al centro di diversi lavori, di taglio locale e nazionale [Vidotto, Tobia, Brice 1998; Franzolini 2006; Labanca 2010; Winter 2014; Bracco 2015]. Molto diverso è il caso della Resistenza, la cui natura di guerra civile non si attaglia alla celebrazione di miti unificanti, come era stato dopo il primo conflitto con figure come la mater dolorosa e il milite ignoto [D’Amelia 2005; Isnenghi 2005].

\footnotetext{
1 Si segnalano in particolare: Galmozzi 1986; Finocchi 1999; Dogliani 2006; Dogliani 1995.
} 
Mentre l'esperienza del primo conflitto vede come protagonista il soldato in armi, il secondo si apre a una moltitudine di testimoni, e anche la stessa Resistenza si presenta come una categoria che trova declinazione in diversi atteggiamenti, comportamenti, azioni.

A ciò si deve aggiungere una certa diffidenza degli ambienti resistenziali per retoriche celebrative e miti patriottici, retaggio del bagaglio culturale fascista da lasciarsi definitivamente alle spalle.

Una proclamata volontà di "smonumentalizzazione" [Poli 1990] che però non impedisce, già all'indomani della Liberazione, di avviare la costruzione di un canone resistenziale, attraverso celebrazioni, memorialistica, monumenti ai caduti e anche attraverso alcune statue.

L'ultimo censimento nazionale dei monumenti alla Resistenza, effettuato dall'architetto Luciano Galmozzi nel 1986, sebbene datato e lacunoso, permette di disporre di dati quantitativi e qualitativi di un certo respiro. Situa l'Emilia-Romagna al secondo posto con 49 opere schedate, in un quadro generale in cui i monumenti sono - prevedibilmente - sparsi sul territorio nazionale rispecchiando fedelmente la geografia della Resistenza. Se esistono diversi monumenti al partigiano, in nessuno dei casi registrati si tratta di partigiani "in carne e ossa", riprodotti nelle loro reali fattezze, ma di trasfigurazioni e rappresentazioni simboliche. Una scelta sulla quale pesa probabilmente anche la volontà di erigere "monumenti a" e non "monumenti di", una sorta di pudore, mosso dal timore di incentivare culti o personalismi.

In questo quadro il caso piacentino presenta alcune peculiarità, per la presenza sul territorio di almeno tre statue che raffigurano comandanti partigiani locali, realizzate dallo scultore Secondo Tizzoni. Non monumenti o mausolei per i caduti - forma più comune di commemorazione del primo periodo postbellico - ma statue figurative, che presentano le fattezze del Valoroso, di Paolo e soprattutto di Emilio Canzi, antifascista di lunga data e comandante unico della XIII zona partigiana. Quest'ultima statua si presenta come un caso eccezionale, per il rilievo della figura rappresentata e il grado ricoperto, e per i meccanismi di costruzione memoriale che ruotano intorno al monumento.

La scelta "controcorrente" dello scultore Secondo Tizzoni, che ritrae i partigiani piacentini con grandissima attenzione alla fisionomia e alla caratterizzazione, permette di toccare alcune questioni più generali circa le forme della memoria. Attorno a statue così particolari ed evocative, si sono concentrate commemorazioni, cerimonie, discorsi pubblici, portati avanti da attori diversi, istituzionali e informali.

\section{La guerra è finita. Smobilitazione e memoria nella "città dei cavalli"}

Il 5 maggio 1945 il centro storico di Piacenza è teatro di un imponente corteo partigiano. È la sfilata della smobilitazione, ultimo grande momento della Resistenza 

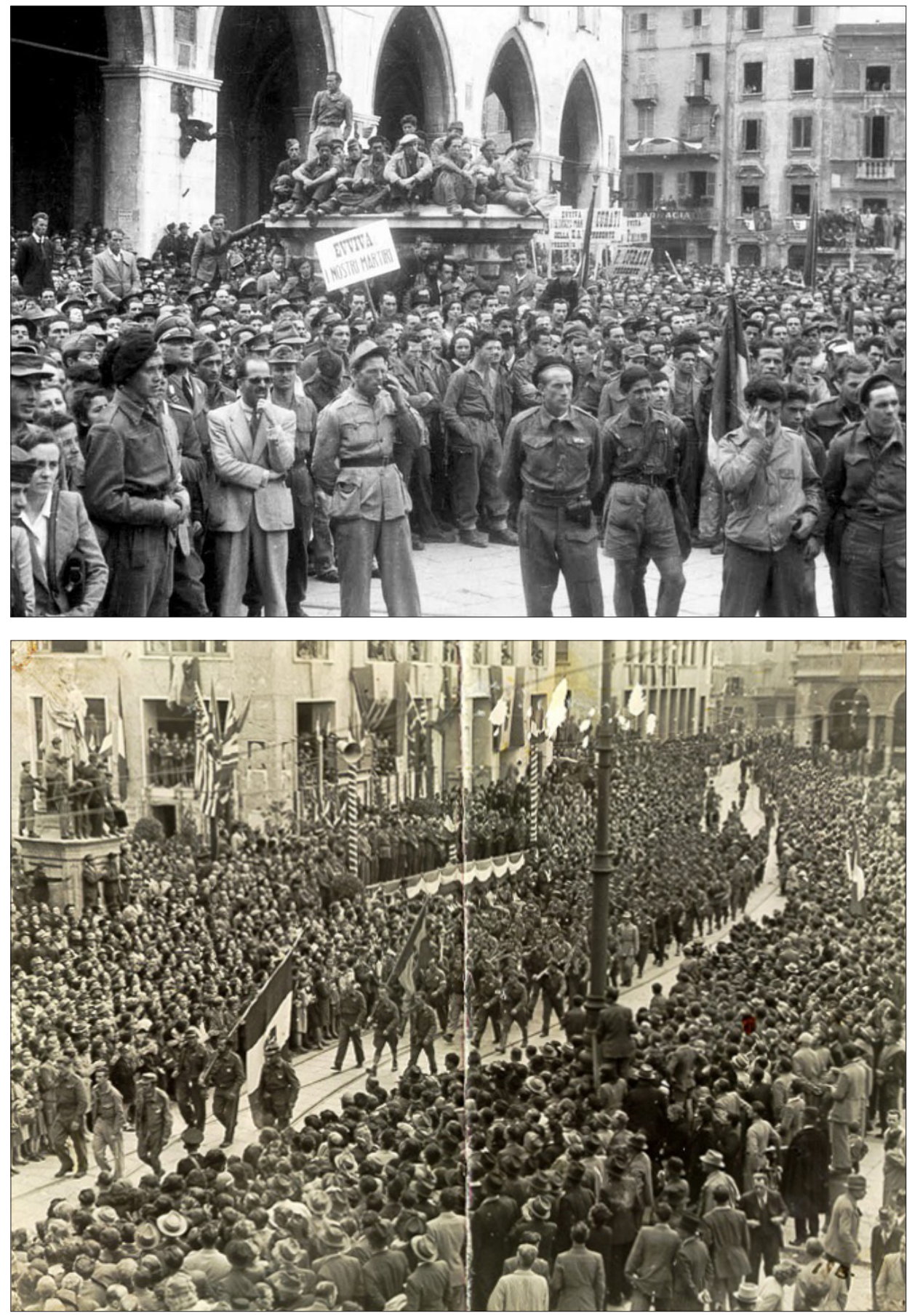

Figg. 2-3. Piacenza, 5 maggio 1945. Sfilata della smobilitazione [Archivio Anpi Piacenza, Fondo Comando Unico]. 
in armi [Meloni 2019, 17-19]. In una città gremita di folla, accorsa a rendere ai partigiani «gli onori degni di tutto il loro luminoso, arduo, faticoso passato, intessuto di indicibili sofferenze» le tre divisioni - prima la Piacenza, poi la Valdarda e infine la Valnure - sfilano, coi loro comandanti in testa, seguite da nuclei di sappisti in bicicletta. In piazza Cavalli, «cuore pulsante della nostra città», da una tribuna «pavesata di bandiere tricolori, alleate e dell'Urss», i partigiani ascoltano il discorso del generale Bowman².

È una scena che si ripete con tempi diversi in tutte le province dove la Resistenza aveva combattuto. Grandi sfilate come quelle di Piacenza rispondono alla necessità pratica - centrale per la Commissione alleata nella delicata fase post-insurrezionale - di procedere al disarmo delle formazioni partigiane nel modo più ordinato e indolore possibile. In questo quadro, la consegna dei diplomi, i discorsi di ringraziamento e stima, specchio di sincero apprezzamento degli angloamericani per la prova di un movimento partigiano «fiorito molto velocemente in quella che sembrava una terra brulla», e «secondo solo a quello jugoslavo» [MacKenzie 2000, 546], sono anche un modo di "indorare la pillola” e segnare pubblicamente la cessazione definitiva del conflitto e delle violenze [Coles, Weinberg 1964, 548; Piffer 2010, 215-238].

In una cerimonia densa di valenze simboliche, i partigiani piacentini sono chiamati a posare a terra, ai piedi degli ufficiali alleati e italiani, le proprie armi, formando - come racconta il comandante di brigata Giuseppe Panni detto Pippo - «cumuli [che] crebbero, fino a diventare altissimi» [Panni 1978, 270].

Nelle foto di quella giornata epocale colpisce una mancanza. In piazza Cavalli, fulcro della manifestazione, non c'è alcun cavallo. Le due grandi statue equestri raffiguranti Alessandro e Ranuccio, realizzate da Francesco Mochi nel XVII secolo per celebrare i duchi Farnese, sono infatti state rimosse, e "sfollate" in campagna. Sentiti come un importante simbolo della città, i "cavalli di Piacenza” vengono in un primo momento protetti da eventuali bombardamenti con delle strutture in legno, poi smontati e portati via [Concarotti 1984].

In occasione della sfilata della smobilitazione del 5 maggio 1945 i basamenti delle statue, vuoti, diventano un punto rialzato di osservazione nella piazza gremita, e gruppi di partigiani vi si arrampicano, e da lì seguono sfilate e cerimonie. Immortalati da scatti fotografici diventano una sorta di icona dell'esplosione incontenibile di entusiasmo di quella giornata.

In un recente volume, Tomaso Montanari ha suggerito una lettura molto evocativa di quelle foto. Scrive lo storico dell'arte: «I piedistalli sono vuoti: non ci sono più padroni. E al posto dei padroni, un nuovo sovrano è salito su quei monumenti: quel sovrano è il popolo [...]» [Montanari 2020, 17].

Certamente i venti mesi della lotta di liberazione rappresentano il processo di costruzione di una nuova sovranità popolare. Se prendere le armi e andare in

2 Le invitte eroiche formazioni patriote passate in rassegna da una Commissione Militare del Quartier Generale del Maresciallo Alexander, in «Piacenza Nuova», 6 maggio 1945, p. 2. 


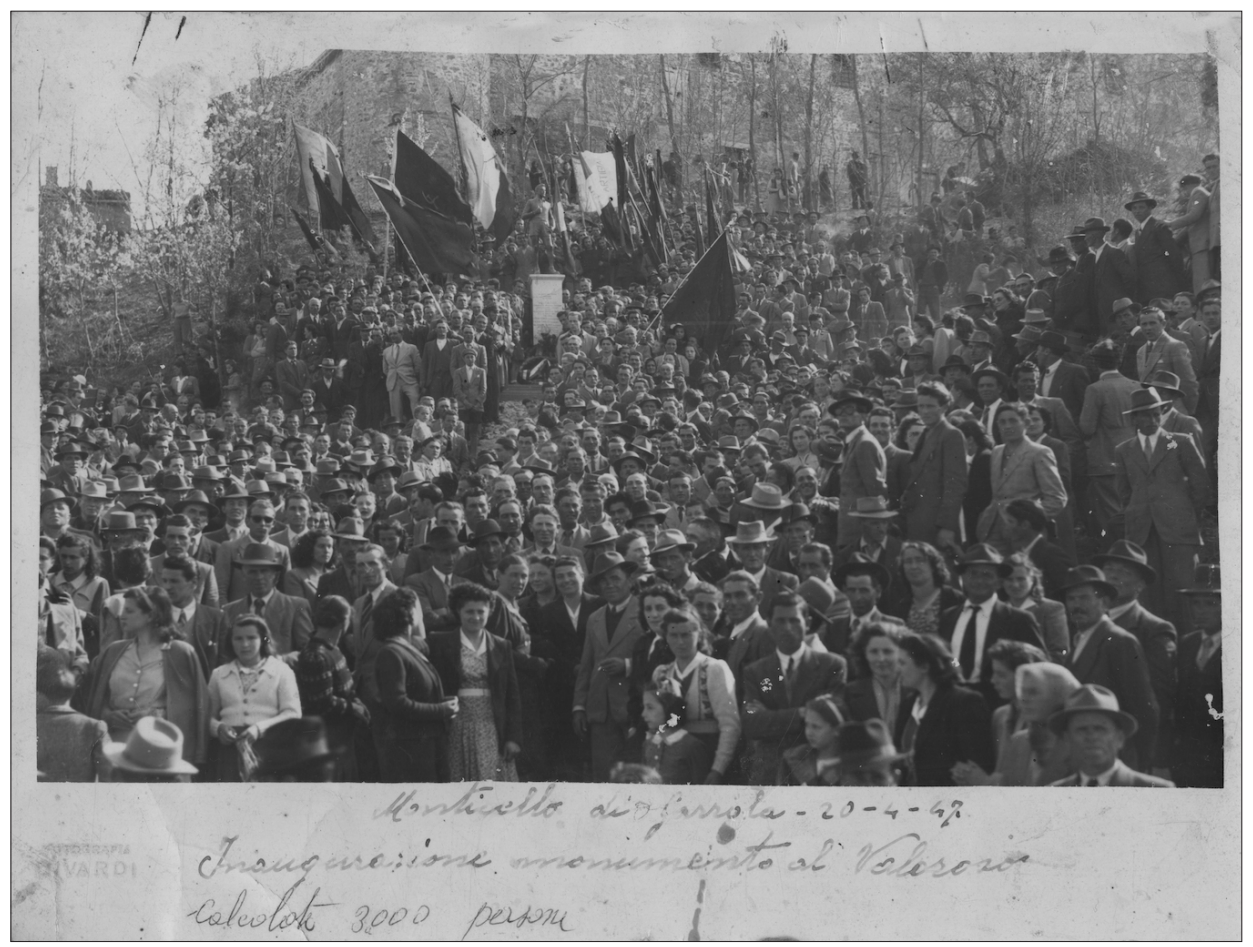

Fig. 4. Monticello di Gazzola, 20 aprile 1947. Inaugurazione della statua di Lino Vescovi Valoroso [Archivio privato famiglia Agosti].

montagna aveva voluto dire per i partigiani riappropriarsi della sovranità individuale, dopo il crollo dello Stato dell'8 settembre 1943, deporre le armi voleva dire rinunciare a quel potere, cederlo a nuove istituzioni, sottoscrivere un nuovo patto sociale [Filippetta 2018].

Anche nella loro assenza forzata i cavalli sanno caricarsi di significati profondi e rimangono uno dei simboli identitari della città. Così fanno anche le statue dei partigiani delle quali ricostruiremo la vicenda, che continuano ad avere ancora oggi un valore memoriale non indifferente. Un tentativo di "scolpire la memoria" che muta nel tempo e che prende il via all'indomani della imponente sfilata del 5 maggio 1945 quando - posati i fucili - comincia il difficile processo di elaborazione del lutto, di ricucitura degli strappi e di ripensamento su quei venti mesi drammatici e coinvolgenti.

\section{3. "Gli eroi son tutti giovani e belli». La statua del Valoroso a Monticello di Gazzola}

Il 20 aprile 1947, a Monticello di Gazzola, una località remota dell'Appennino piacentino, viene inaugurata la statua dedicata a Lino Vescovi, detto Valoroso, realizzata dallo scultore Secondo Tizzoni. Il luogo scelto è quello dove, due anni 
prima, il comandante della IX brigata era morto, colpito durante un imponente combattimento nel quale un gruppo di partigiani aveva avuto la meglio su più numerosi contingenti fascisti.

Classe 1920, il Valoroso, nativo della "bassa", era salito in montagna nella primavera del 1944, e si era imposto come un capo carismatico e audace - valoroso appunto - ricordato nella memorialistica come generoso e abile nei colpi di mano. A renderlo leggendario sono le circostanze della sua morte, e la grande tenacia testimoniale della sorella e compagna di lotta Alberta, che per tutta la vita porta avanti la memoria del fratello, trasmettendo fortemente al figlio il ruolo di "candela della memoria” del giovane partigiano ucciso [Wardi 1993; Agosti, Vescovi 2021]. Anche il fatto di essere una delle ultime vittime della lotta di liberazione colpisce l'attenzione di un movimento partigiano "imponente", che ormai non è più composto di piccoli gruppi sparsi ma di divisioni e brigate. Quando il Valoroso muore, la Resistenza piacentina dispone ormai di organi di stampa, dai quali, da subito, si comincia a costruire l'epica della battaglia del Monticello e dei suoi morti.

Già nel 1946 il castello di Monticello, fulcro della battaglia, è luogo di partenza di una grande manifestazione pubblica organizzata dall'Anpi (Associazione nazionale partigiani d'Italia). In una vera e propria processione, la salma del Valoroso era stata traslata dal cimitero di Piozzano per attraversare Piacenza issata su un fusto di cannone e ricevere gli onori della folla, e infine essere trasportata a Monticelli d’Ongina, paese natale del partigiano.

Sulla sua tomba viene posta una prima statua in bronzo, sempre opera di Tizzoni, dove il partigiano imbraccia lo sten (che nella versione del 1947 porterà invece in spalla).

Il quotidiano locale sottolinea come la statua di Tizzoni intenda trasporre in bronzo proprio la personalità del Valoroso, che «prima di diventare un eroe, fu un uomo». Sia nella statua posta al cimitero che in quella innalzata sul luogo della morte il partigiano

non era più che un ragazzo, un minuscolo ragazzo tutto nervi ed energia, che aveva tre grandi passioni: i motori, le armi e la musica [...]. Il suo vero nome era Lino Vescovi: un piccolo italiano qualsiasi che in epoche normali non sarebbe stato forse altro che un buon meccanico specializzato. Oggi è il Valoroso, simbolo di un'idea e materia di una leggenda per quelli che verranno ${ }^{3}$.

Vale la pena di notare che di occuparsi del coordinamento della laboriosa operazione è incaricato da subito Ludovico Muratori, Muro, comandante partigiano che aveva preso parte alla battaglia del Monticello, e - lo vedremo meglio in seguito - vero e proprio tessitore paziente della memoria della Resistenza piacentina, anche attraverso i linguaggi dell'arte ${ }^{4}$.

3 Questo il Valoroso, in «Libertà», 15 aprile 1946, p. 1.

4 Archivio Anpi Piacenza (ANPIPC), Amministrazione, Verbali 1945-1963, b. 3, verbale 8 aprile 1946. 


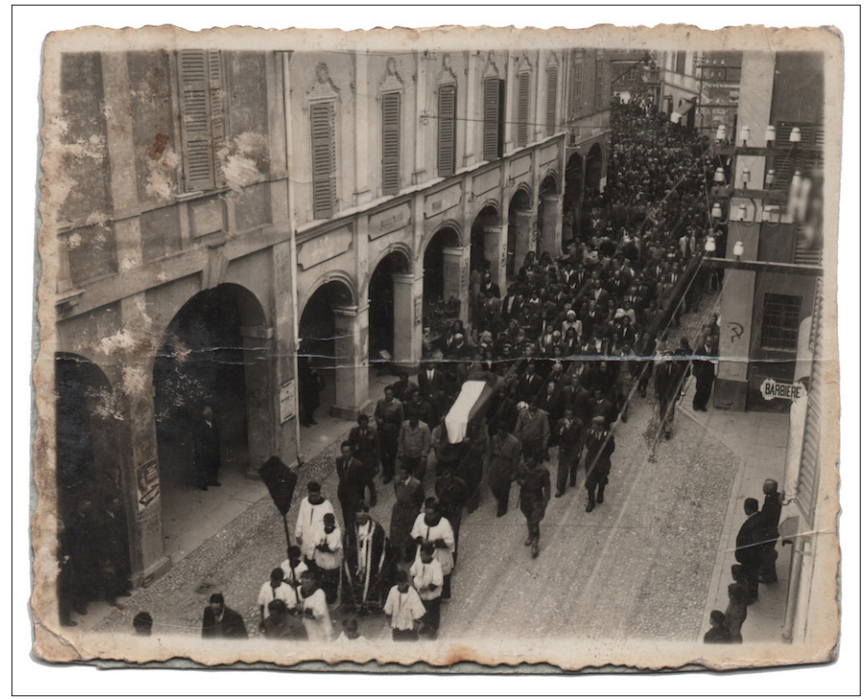

Fig. 5. Monticelli d'Ongina, 15 aprile 1946. Traslazione della salma di Lino Vescovi [Archivio privato famiglia Agosti].

Il grande funerale pubblico del Valoroso è solo una delle imponenti cerimonie funebri che attraversano l'Italia in quegli anni, colpita dalla presenza pulsante, "viva", dei caduti, e impegnata a elaborare il lutto soprattutto attraverso il comandamento morale di onorare il "popolo dei morti” [Paggi 2009; Schwarz 2010]. Già nell'agosto 1945 il preoccupato segretario della neonata sezione Anpi di Piacenza relazionava al presidente, il comandante Canzi, che «la situazione finanziaria è tutt'ora in passivo per le forti spese incontrate per funerali, esumazioni e trasporto salme dei partigiani caduti» ${ }^{5}$.

L'attività di esumazione delle salme (spesso sepolte provvisoriamente nei cimiteri di montagna, e che si sente in qualche modo di dovere "portare a casa") e l'apposizione di cippi nei luoghi di uccisioni e eccidi, impegna in modo notevole l'associazione, e culmina nella costituzione di una commissione specifica. Formata da tre noti comandanti partigiani (Ludovico Muratori, Fausto Cossu e Carmelo Giuffrè), la commissione tratta dei prezzi del marmo con le cave, si preoccupa degli inviti da inviare per le inaugurazioni dei vari cippi, e ragiona sulla possibilità di erigere presso il cimitero di Piacenza un monumento a tutti i caduti. Si pensa di utilizzare una parte della colonna del monumento della Lupa, eretto nel 1938 per commemorare la proclamazione dell'Impero ${ }^{6}$, o di monumentalizzare una parte del muro dove erano stati fucilati per rappresaglia diversi partigiani e prigionieri politici ${ }^{7}$. Si tratta infine con il Comune la concessione perpetua di un

5 Ivi, verbale 8 agosto 1945.

6 ANPIPC, Attività, Erezione cippi e monumenti, b. 112, Lettera di Giovanni Grcavaz al sindaco di Piacenza, 21 febbraio 1947, Monumento partigiani caduti.

7 Ivi, Lettera del Comitato provinciale Anpi al sindaco di Piacenza, 15 maggio 1947, Sistemazione cinta murale cimitero urbano. 
Fig. 6. Monticelli d'Ongina, 15 aprile 1946. Inaugurazione della statua di Lino Vescovi Valoroso al cimitero [Archivio privato famiglia Agosti].

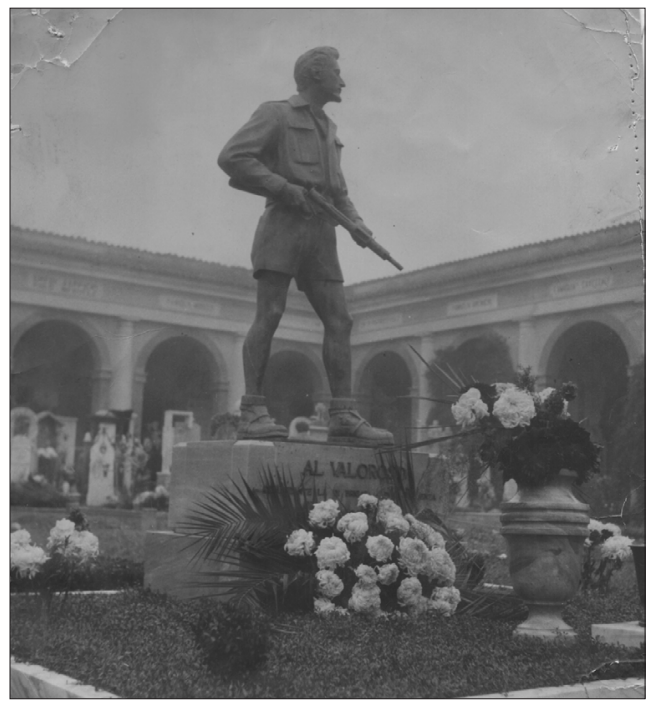

pezzo di terreno da adibire a "cimitero partigiano". Su questo si apre anche una singolare querelle interna all'associazione, che rivela l'incertezza di questa prima fase commemorativa. Per alcuni infatti il cimitero partigiano è troppo piccolo, e non vi troveranno spazio «tutti i partigiani che, oltre ai caduti nella lotta di Liberazione, moriranno nel comune di Piacenza» ${ }^{8}$. Per provvedere alla manutenzione dei cippi e all'organizzazione delle commemorazioni si forma anche l'Associazione famiglie partigiani caduti, guidata da Maria Carella Baio, madre di Cesare, giovane antifascista deportato?.

In un clima di grande attenzione al culto dei "martiri della Resistenza”, la figura del Valoroso, giovane partigiano in armi, ben si attaglia alla memoria pubblica militare, combattentistica, quasi epica - della lotta di liberazione nei primissimi anni del dopoguerra, e la statua di Tizzoni traduce perfettamente questa idea, immortalando i tratti del comandante, ritratto con scarponi da montagna e pantaloni corti, come nelle poche fotografie da partigiano.

Vale la pena mettere a confronto questa scultura con quella che forse è la prima statua di partigiano di una certa rilevanza artistica, il Palinuro o monumento al partigiano Masaccio scolpita da Arturo Martini. L'opera è commissionata dai compagni di brigata di Primo Visentin, Masaccio, partigiano e studioso di arte, ucciso nel trevigiano durante la ritirata tedesca il 29 aprile 1945. La scelta artistica del celebre Martini, scultore di grande esperienza e fama, è diametralmente opposta a quella di Secondo Tizzoni. Mentre Masaccio appare trasfigurato nelle sembianze in Palinuro - eroe della mitologia virgiliana, caduto in mare poco prima che la flotta di Enea si mettesse in salvo - il Valoroso è raffigurato con quella

8 Ivi, Lettera del segretario comunale Anpi Rizzi al sindaco di Piacenza, 30 luglio 1947, Sistemazione tombe partigiani caduti.

9 Ivi, Lettera di Maria Carella Baio al prefetto di Piacenza, 20 maggio 1947, Comunicazione. 
«fedeltà fisiognomica» [Fugazza 2000,3] che contraddistingue Tizzoni e che fa dei suoi lavori un unicum in Emilia-Romagna.

La statua del Valoroso si colloca in una fase di urgenza di commemorazione, che si traduce in genere in cippi e lapidi, posti da famiglie, compagni di battaglia, formazioni partigiane, espressione della volontà di "fissare" e testimoniare perdite e traumi. Una "rivoluzione copernicana”, che sposta l'enfasi monumentale dalle piazze - luogo principe del ricordo dei caduti della Grande Guerra - a sedi periferiche, riconosciute come significative per singole comunità, associazioni, gruppi sociali o politici. In più di un Comune il monumento ai caduti del 1915-18 viene "aggiornato" con i nomi dei caduti partigiani (spesso di tutti i soldati caduti tra 1940 e 1945), «in un patriottico abbraccio cumulativo» [Baioni 2020, 204]. Più rare sono in questa fase le forme artistiche, tra le quali vale la pena ricordare il precoce monumento al partigiano di Imola, inaugurato già nel 1946, che però raccoglie tutti i caduti e li rappresenta in forma di guerriero primitivo e archetipico [Galmozzi 1986, 10] ${ }^{10}$. L’Anpi di Piacenza sceglie di affidare il compito di “scolpire la memoria” a Tizzoni, classe 1916, da poco diplomato all'Accademia di Brera e molto promettente ${ }^{11}$. Il suo legame con la Resistenza viene soprattutto dalla sorella Bruna, attiva agente di un importante servizio di intelligence partigiana alle dipendenze del Comitato di liberazione nazionale Alta Italia [Forlani 1993; Oltremonti 2012]. Alla Liberazione, era stato Lorenzo Marzani, detto Isabella, amico e stretto collaboratore del comandante unico Emilio Canzi a nominare, per conto del Comitato di liberazione nazionale, Secondo Tizzoni alla cattedra di "geometria descrittiva" presso il locale istituto d'arte [Fugazza 2000, 3]. Insomma, anche se non aveva fatto il partigiano, Tizzoni era sicuramente una persona della quale il movimento resistenziale piacentino aveva fiducia, tanto da commissionargli i ritratti dei suoi "eroi".

Nel 1947, contestualmente alla posa del monumento del Valoroso al Monticello, Secondo Tizzoni lavora a un altro ritratto, che modella in creta prima di predisporre una forma in gesso per la fusione in bronzo. Fusione che tuttavia dovrà aspettare ancora fino al 1955. Stavolta infatti la figura che Tizzoni vuole riprodurre è problematica, pungente, e interroga il movimento partigiano in alcuni dei suoi nodi allora ancora irrisolti.

\section{4. «Egli cammina ancora»...}

La statua di Emilio Canzi scolpita da Tizzoni è diventata negli anni il luogo della memoria per eccellenza della Resistenza piacentina, e forse non solo. Meta di

10 Proprio questo monumento è stato in anni recenti al centro di polemiche giornalistiche relative alla riconversione della statua, che secondo esponenti dell'estrema destra avrebbe rappresentato - nelle reali intenzioni del suo autore - i caduti della guerra d'Etiopia. Un'ipotesi fondata sostanzialmente - nella dichiarata mancanza di documentazione a riguardo - su un bassorilievo sul basamento raffigurante un'antilope.

11 «Piacenza Nuova», 22 maggio 1945. 
Fig. 7. Emilio Canzi [Archivio Gruppo ricerca immagine, Bettola].

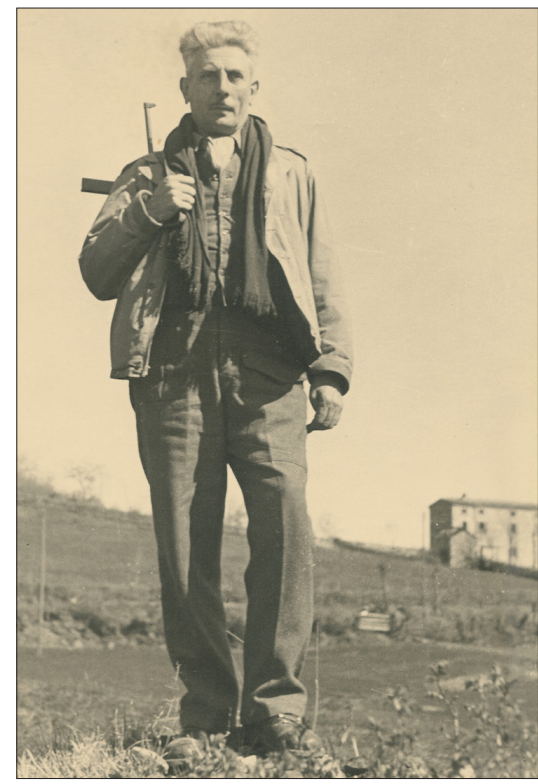

pellegrinaggi e commemorazioni, la statua del comandante - posta in montagna, a Peli di Coli, dove Canzi aveva combattuto - è oggi il luogo di incontro di generazioni e memorie molteplici, centrali e locali.

E questo può in un certo senso stupire se pensiamo alla storia dell'anarchico Canzi, una storia tutt'altro che pacificata e "condivisa”.

A cavallo tra Lombardia ed Emilia-Romagna, provincia di confine e dalla doppia natura, Piacenza diviene nel corso della lotta di liberazione epicentro di alcune linee di tensione interne al movimento resistenziale. La sua importanza strategica e politica ne fa una provincia contesa, dove contrasti che attraversano tutta l'Italia partigiana esplodono in episodi drammatici e feroci [Dondi 2004]. È il caso del gruppo di partigiani guidati dal comunista Giovanni Molinari, Piccoli, ucciso nel giugno 1944 da una banda di carabinieri, per ragioni legate al controllo del territorio. A partire da questo episodio, due delle quattro valli piacentine saranno terreno di azione della più folta divisione partigiana piacentina, la Piacenza legata (per ragioni di copertura politica prima che di adesione ideale) al movimento giellista [Sprega 1998]. Ed è anche il caso dell'anarchico Canzi. Si tratta ancora di una questione che vede contrapporsi centri di potere diversi: Comando Nord Emilia (Cne) e Comitato di liberazione nazionale (Cln), Partito comunista e Partito d'azione, Milano e l'Emilia. Nato a Piacenza nel 1893, Emilio Canzi è antifascista di lunga data, ardito del popolo, combattente della guerra civile spagnola e infine comandante unico dei partigiani piacentini [Silingardi 2003]. Nel corso della Resistenza e nell'immediato dopoguerra proprio questa carica apicale, di alto valore militare e simbolico, è al centro di un conflitto acceso e doloroso. A partire dall'inverno 1944-45, quando un grande rastrellamento scompagina completamente le formazioni, il Partito comunista cerca infatti di rimediare all'assenza di uomini in posti di comando in provincia. Una volontà che si salda con le critiche da più parti rivolte a Canzi per 
non aver saputo contrastare il disastro del rastrellamento, e che mette in discussione - come accade in altre province - la reale efficacia militare degli organismi di raccordo e coordinamento della Resistenza.

In un crescendo di accuse, di diffidenze, di recriminazioni, all'inizio dell'aprile 1945 il Cne sostituisce il comando unico "pluralista" guidato da Canzi con uno "monocolore” guidato da Luigi Marzioli, Marzi. All'interno di un infuocato conflitto di legittimità tra Cne e Comando generale del Corpo volontari della libertà su chi avesse la facoltà di procedere effettivamente alla nomina, Canzi subisce la vergogna del fermo operato da uomini della divisione Valnure e dell'imprigionamento in una casa privata. Riesce comunque a essere liberato e a partecipare alla presa della città, pur senza il grado di comandante.

Segue la sfilata della smobilitazione del 5 maggio dal palco delle autorità, fianco a fianco con coloro che ne avevano ordinato l'arresto. Non riceve nessun diploma, ma significativi applausi dai suoi partigiani. Nelle cronache della giornata è indicato come «Comandante unico della montagna», termine ambiguo che maschera, sotto una narrazione unitaria e trionfalistica, l'insolita presenza in piazza Cavalli di due comandanti unici.

Nel dopoguerra, pur rispettando le esigenze della pacificazione, Canzi da una parte si batte per la riconciliazione e la pace, ma dall'altra, appoggiato dal Partito d'azione, ribadisce con tenacia l'ingiustizia della quale era stato vittima. Il Comando generale di Milano apre un'inchiesta, mentre i comunisti continuano a giustificare con la presunta inettitudine militare di Canzi il colpo di mano che aveva portato alla nomina di Marzioli. Il 5 novembre 1945 l'inchiesta giunge a termine con una soluzione di compromesso: pur non sconfessando la nomina di Marzioli, il Comando generale riconosce a Canzi la qualifica di comandante unico dalla formazione del comando stesso alla smobilitazione. A sbloccare la situazione è forse anche una terribile e paradossale fatalità: il 30 settembre Canzi è stato vittima di un incidente stradale, e ormai versa in condizioni critiche. Il comandante unico fa comunque in tempo ad apprendere la bella notizia prima di morire, il 17 novembre 1945 [Silingardi 1991]. A distanza di settant'anni da quel tragico incidente la morte di Canzi, così "provvidenziale” per i suoi avversari, è ancora al centro di polemiche, accuse, domande aperte.

Il funerale si celebra quattro giorni dopo nella chiesa di San Francesco, per volere della famiglia, nonostante secondo la cronaca locale diversi «comunisti libertari» chiedano che «coerentemente con le idee del defunto» le esequie siano celebrate in forma civile. A officiare la cerimonia don Giovanni Bruschi, cappellano militare della XIII zona, parroco di Peli, amico e collaboratore fidato di Canzi nella Resistenza. Dopo la cerimonia e i discorsi ufficiali, un imponente corteo accompagna «il Comandante Canzi che ritorna in montagna, dalla quale era sceso, per restarvi come nume tutelare» ${ }^{12}$. Per sua espressa volontà, Emilio Canzi viene sepolto a Peli di Coli, dove era cominciata la sua attività da partigiano.

12 Gli imponenti onori funebri tributati ieri a Canzi, in «Libertà», 22 novembre 1945, p. 1. 
Fig. 8. Santino funebre di Emilio Canzi [Archivio Anpi Piacenza, Fondo Schede Anagrafiche].

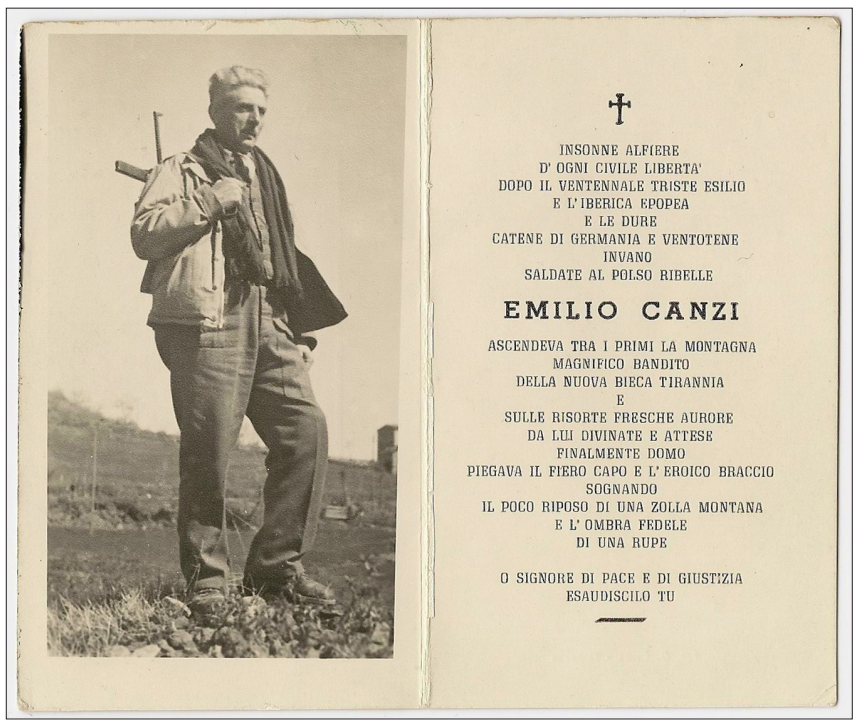

Il conflitto per il comando unico riverbera nelle memorie, crea spaccature e silenzi, e rappresenta in molta parte della memorialistica sulla Resistenza piacentina un rimosso, un oblio in qualche modo necessario alla pacificazione. Una memoria dolorosa, molto diversa da quella unanimista del Valoroso, che forse spiega perché la statua di Secondo Tizzoni - ideata già nel 1947 - viene posta a Peli solamente il 24 luglio 1955. Ad occuparsi della cerimonia è un comitato creato ad hoc, che raccoglie istituzioni, associazioni e amici, e che commissiona la scultura e la posa di una lastra di marmo con epigrafe sulla tomba del comandante. Forse il clima generale del recente decimo anniversario della Liberazione, che ha dato vita anche a livello nazionale a un rilancio della memoria resistenziale, aiuta a sbloccare la situazione. A livello provinciale vengono realizzati una serie di progetti, pubblicazioni storiche, mostre, e viene avviato il processo per il conferimento della medaglia d'oro per la città ${ }^{13}$ [Morsia 2001].

Nelle celebrazioni ufficiali del 25 aprile 1955 l'attenzione non è più rivolta ai caduti ma si apre ad altri soggetti della lotta di liberazione. In piazza Cavalli, il piacentino Doro Lanza, detto Carlo Martello, afferma esplicitamente:

Non vogliamo oggi nominare nessuno dei morti, degli innumerevoli morti [...]. Vogliamo soltanto rivolgere il pensiero agli umili operai, ai contadini, alla gente semplice alla quale forse nessuno aveva spiegato il significato della Resistenza ma che seppero tacere nei tormenti, che combattendo e morendo si sentirono improvvisamente fratelli ${ }^{14}$.

13 Archivio di Stato di Piacenza (ASPC), Fondo Cvl, b. 3, fasc. 1, Comitato per le celebrazioni.

14 Il corteo con i gonfaloni dei Comuni e il discorso celebrativo dell'onorevole Mazza, in «Libertà», 26 aprile 1955, p. 2. 
Proprio in montagna, alla presenza di «numerosi valligiani della zona», viene posta la statua di Canzi, in un luogo evocativo e antiretorico, in posizione avanzata, su un piedistallo in pietra, con lo sguardo rivolto all'immensità della valle. Una volta scoperta la scultura, a parlare sono Fausto Cossu, e Leonida Patrignani, Bandiera, già capo partigiano delle formazioni gielliste piacentine e militante del Partito d'azione fino al suo scioglimento ${ }^{15}$. Nessun comunista prende la parola, e a leggere le cronache di quel giorno, le uniche presenze riconoscibili sono quelle anarchiche e gielliste.

Come aveva già fatto per il Valoroso, nel realizzare la scultura di Emilio Canzi, Secondo Tizzoni lavora sulle fotografie per raggiungere una fedeltà ritrattistica ${ }^{16}$. Il modello è sicuramente dato da una serie fotografica di grande valore - della quale non è stato possibile finora individuare con certezza l'autore - che per la sua comunicatività è divenuta simbolo della Resistenza piacentina. Il profilo del comandante unico si staglia su uno sfondo brullo e anonimo, che suggerisce un indistinto scenario della collina teatro della lotta partigiana. Rappresentato come partigiano semplice, Emilio Canzi, con i capelli bianchi, si protende in una posa di grande plasticità. Il passo accennato evoca un movimento, un percorso di militanza che viene da lontano e che va lontano. Lo sten, simbolo di una lotta combattuta, è in spalla e richiama la calma, la serenità della giustizia.

Una versione autografata permette di collocare lo scatto nel luglio 1945.

In quel momento Emilio Canzi sta rivendicando con forza l'ingiustizia della sua sostituzione. Contro questa decisione aveva presentato un ricorso, e proprio nel luglio si era recato diverse volte a Milano per conferire con esponenti del Partito d'azione e perorare la sua causa. In quella contesa aperta, l'icona fotografica lancia un messaggio di incredibile potenza comunicativa. Traduce in immagine alcune delle ragioni di Canzi. La militanza di lunga data, i «venticinque anni di lotta, di sacrifici senza limiti [...], il tormento del freddo e della fame, la preoccupazione della morte vicina», che gli conferiva una autorevolezza che Marzioli, «entrato ora, cinque minuti prima della fine, per raccogliere i frutti delle fatiche altrui, non aveva». Il carisma e la credibilità diffusi presso la base, che derivavano dall'aver affrontato fianco a fianco con i propri uomini la lotta partigiana, che facevano di Canzi una figura realmente rappresentativa, al contrario di Marzioli, con la fama di imboscato e di partigiano dell'ultima ora [Silingardi 1991, 31; Dondi 2004, 367-373]. Nella sua semplicità, la foto afferma con la forza delle immagini l'autorevolezza assoluta, quasi paterna, di Canzi, e ribadisce il concetto che c'è un comandante vero e uno che invece è tale solo sulla carta, in virtù di un sordido «complotto politico» ${ }^{17}$.

15 Il monumento a Canzi inaugurato a Peli, in «Libertà», 25 luglio 1955, p. 4.

16 La particolare sensibilità ritrattistica di Tizzoni è suggerita anche dalla scelta, inconsueta, di ritrarre nelle litografie di nudo le modelle con una particolare fedeltà ai tratti del volto.

17 Emilio Canzi “Ezio Franchi”, a Comando generale Alta Italia, 7 aprile 1945, in Silingardi 1991, 30-31. 
Fig. 9. Emilio Canzi, ritratto fotografico con dedica a Giovanna Berneri [Archivio Famiglia Berneri - A. Chessa].

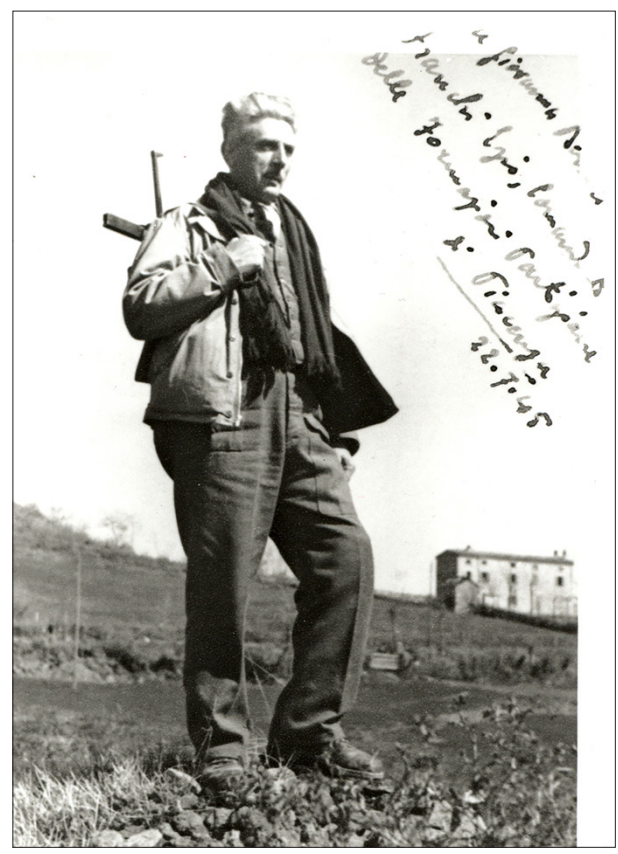

La sapienza compositiva e l'evidente scopo morale/pedagogico dello scatto, consentono di ipotizzare che per l'occasione fosse stato chiamato un professionista, magari proprio quel Gianni Croce che, a distanza di pochi mesi, compone il santino funebre ${ }^{18}$. Anche se le lastre originali non sono conservate presso l'archivio del fotografo, a rafforzare questa supposta attribuzione contribuiscono l'abilità ritrattistica, l'uso della luce, il taglio dell'inquadratura, la capacità di riproduzione del movimento [Bonvicini 2002; Cavalloni, Panciroli 2016]. In occasione degli imponenti funerali di Canzi la foto viene riprodotta in numerosi manifesti e foto ricordo $^{19}$, e sui giornali con una epigrafe incredibilmente antiretorica e che rende ancora più esplicito il messaggio:

Egli cammina ancora

come ha camminato tutta la vita

incontro ai suoi fantasmi luminosi e inquieti.

Cammina sulla montagna di Peli, ed è sereno.

Altri, intorno a lui, camminano con lo stesso silenzioso suo passo ${ }^{20}$.

18 ANPIPC, Fondo Schede Anagrafiche, b. CA-CAP.

19 ANPIPC, Amministrazione, Verbali 1945-1963, b. 3, verbale 23 novembre 1945.

20 «Libertà», 23 novembre 1945, p. 1. 
Concetti presenti - con toni invece intrisi di retorica - anche nell'iscrizione del santino funebre, dove si richiamano la «iberica epopea», le «catene di Germania e di Ventotene invano saldate al polso ribelle» di Canzi, «magnifico bandito». Entrambe le iscrizioni hanno lo scopo di

riportare l'attenzione sulle culture politiche minoritarie che durante la dittatura avevano tenuto in vita - per vie clandestine, nell'esilio, con le brigate internazionali nella Guerra civile spagnola - la tradizione di libertà e giustizia risalente al filone democratico del Risorgimento; e dimostrare come tali rivoli fossero confluiti in forma originale nella partecipazione popolare nella guerra partigiana [Baioni 2020, 202-203].

Quella serie di fotografie, che la commozione per la morte di Canzi rendono ben presto iconiche, Secondo Tizzoni la riproduce fedelmente in creta, poi in gesso e infine in bronzo. Sicuramente l'equilibrio compositivo colpisce lo scultore e ben si sposa con il suo stile, in cui l'arte figurativa ripudia l'enfasi alla ricerca di una «dimensione classica, in cui regnano l'armonia e l'equilibrio delle forme» [Fugazza 2000, 3]. Lo colpiscono a tal punto da allineare anche la statua del Valoroso nella sua seconda versione, quella posta a Monticello di Gazzola nel 1947, a quel canone compositivo di straordinario impatto visivo.

Un'idea feconda se, da allora fino a oggi, quelle statue hanno saputo far parlare davvero i partigiani, raccontare la loro storia ad altre generazioni, e sono state teatro di notevoli pratiche commemorative.

\section{5. «Prima che nel bronzo egli era scolpito nel nostro spirito». Gli anni Sessanta e Settanta}

Ormai riconosciuto come "scultore ufficiale" della Resistenza piacentina, Secondo Tizzoni viene ingaggiato per ritrarre un terzo partigiano. Il 23 ottobre 1960, dopo una lunghissima querelle circa la collocazione, viene inaugurata a Rivergaro (Piacenza) la statua a Alberto Araldi, Paolo, vero e proprio simbolo della Resistenza in Val Trebbia [Meloni, Menzani 2019, 64-67]. Catturato nel corso di un'azione gappista e fucilato dopo un processo sommario il 9 febbraio 1945, Paolo viene raffigurato come «un giovane slanciato, ignudo e con le mani legate, che sembra staccarsi da terra per protendersi verso il cielo». A curare l'erezione del monumento, a nome del Consiglio federativo della Resistenza che riunisce le diverse associazioni partigiane, è ancora una volta Ludovico Muratori, che stavolta lavora fianco a fianco con Italo Londei, capo partigiano della Val Trebbia. L'orazione ufficiale è tenuta dal comandante di Araldi, Fausto Cossu, che ammonisce che «questo monumento non aggiunge nulla alla gloria di Paolo, perché prima che nel bronzo egli era scolpito nel nostro spirito» ${ }^{21}$.

21 ANPIPC, b. 112, Erezione cippi e monumenti, fasc. Monumento a Paolo. 
Negli anni successivi numerosi monumenti vengono eretti in tutta la provincia per ricordare i caduti partigiani. Nel 1963 viene scoperto a Tabiano di Lugagnano il busto di Wladimiro Bersani, capitano Selva, comandante garibaldino lì ucciso in combattimento nell'aprile 1944. Realizzata da Attilio Bersani, figlio del caduto, la scultura viene inaugurata alla presenza di numerose autorità e di due partigiani che erano presenti alla morte del comandante ${ }^{22}$. Anni dopo, il busto, abbattuto da un atto vandalico, sarà al centro di una lunga campagna stampa relativa ai valori della Resistenza e alla cura di cippi e monumenti ${ }^{23}$.

Nell'aprile 1969 viene inaugurato il cippo dedicato a Rino Cavaliere, a Montegiogo di Lugagnano, e nel novembre successivo in località Cave di Albarola il monumento dedicato a Archibald Donald Mackenzie, scozzese, prisoner of war detenuto nel campo di Veano e comandante partigiano in Val Nure [Hullis 2014]. Alla cerimonia prendono parte due vice-consoli in rappresentanza del governo britannico ${ }^{24}$.

Gli anni Settanta sono anni di rilancio della Resistenza e di riscoperta della storia partigiana, da parte soprattutto delle giovani generazioni. Protagonisti di questa stagione sono, a Piacenza, i ragazzi del Comitato antifascista militante (Cam), attivo tra il 1974 e il 1978.

Il Cam declina in chiave locale temi propri del movimento studentesco di quegli anni: la dimensione di classe della lotta partigiana, il recupero di modelli di democrazia diretta, la visione della Resistenza come "occasione mancata" e rivoluzione interrotta, la critica alle retoriche celebrative delle commemorazioni ufficiali [De Luna 1995, 141-148; Focardi 2005, 46-55]. All'interno di una sofferta riflessione sulla legittimità della violenza politica l'esempio dei partigiani viene messo al centro di dibattiti e riflessioni, e si costruiscono legami di memoria tra la Resistenza e l'antifascismo militante [Gambetta 2000; Matteo 2020].

Nato sulle spinte del presente - la paura di una deriva autoritaria legata alla strategia della tensione, il senso di trovarsi immersi in un «momento di scontro politico molto acuto» in occasione del referendum sul divorzio, la constatazione di continue aggressioni neofasciste - il Cam porta avanti alcune battaglie caratterizzanti, come quella per la messa al bando del Movimento sociale italiano, l'antimperialismo, e l'assunzione di «un ruolo di pungolo nei confronti di altre organizzazioni del movimento operaio e popolare affinché si caratterizzino sempre più in senso antifascista» ${ }^{25}$. L'“antifascismo militante" del Comitato è letto in diretta continuità con la Resistenza, la cui storia diventa «un importante patrimonio di cui armare la nostra lotta al fascismo» ${ }^{26}$, sostenuta anche dalla "constatazione che la politica condotta dalla borghesia e dalla DC [Democrazia Cristiana] tenda in

\footnotetext{
22 Ivi, fasc. Monumento a Wladimiro Bersani.

23 Una presa di posizione delle associazioni partigiane, in «Libertà», 27 agosto 1982, p. 4.

24 ANPIPC, b. 112, Erezione cippi e monumenti, fasc. Monumento Mak.

25 Archivio Comitato antifascista militante (CAM), Elementi di bilancio sull'attività del CAM nel periodo marzo '74-marzo '75, s.d.; Ivi, Presentazione, in «Antifascismo Militante» («AM»), aprile 1974.

26 A30 anni dall'esperienza storica della Libera Repubblica di Bettola, in «AM», dicembre 1974, p. 6
} 


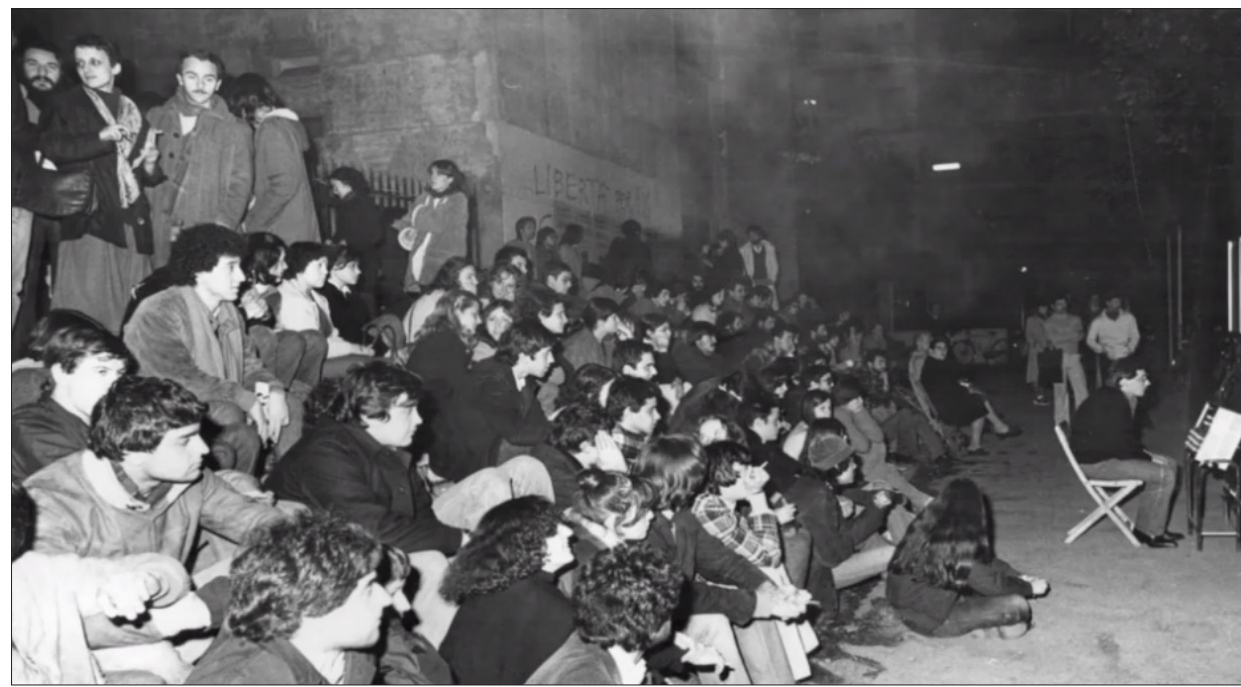

Fig. 10. Veglia antifascista del Cam a Barriera Genova, s.d. [Archivio Comitato antifascista militante].

realtà a negare i valori della guerra di liberazione» ${ }^{27}$. «Antifascismo Militante», bollettino del Cam, pubblica numerosi articoli e inserti sulla storia del fascismo e della Resistenza, lasciando spazio anche a ricerche originali sulla storia loca$\mathrm{le}^{28}$. Il Comitato è protagonista di una stagione di rinnovamento delle pratiche commemorative e delle modalità comunicative, che avvicinano alla storia dei partigiani tanti giovani. Uno spettacolo cantato dal titolo Ora e sempre Resistenza viene messo in scena dalla Commissione artistica del $\mathrm{Cam}^{29}$, si organizza una

27 I GAP e le SAP. Un contributo importante alla lotta partigiana, in «AM», febbraio 1975.

28 In particolare si segnalano: le zone libere dell'estate 1944 (A 30 anni dall'esperienza storica della Libera Repubblica di Bettola, in «AM», dicembre 1974); l'attività delle Squadre di azione patriottica Bertè, Borotti e Oltrepò (I GAP e le SAP. Un contributo importante alla lotta partigia$n a$, in «AM», febbraio 1975); il ruolo delle donne negli scioperi dei bottonifici piacentini e nella Resistenza (Le donne nella lotta antifascista a Piacenza, in «AM», marzo 1975; Ilda l'agitatrice. Testimonianza di Ilda Barbieri "Gneta” di Giustizia e Libertà, ivi; Le donne piacentine e la lotta di liberazione, in «AM», aprile 1978); la lotta degli Arditi del popolo a Parma e Piacenza (Episodi e testimonianze della lotta antifascista, in «AM», aprile 1975); la battaglia di Monticello di Gazzola e la figura del Valoroso (Il Valoroso dà scacco ai nazifascisti, in «AM», aprile 1975); l'eccidio di Rio Farnese (Bettola, il monumento ai partigiani trucidati, in «AM», luglio-agosto 1975); la stampa clandestina (Colpo di mano della propaganda partigiana, in «AM», febbraiomarzo 1976); lo squadrismo in Val Tidone (Cerri: ricordi della lotta antifascista a Borgonovo, in "AM», aprile 1976); la figura di Cesare Rabaiotti, Moro, e le "puntate” sulla Via Emilia (Il Moro terrore della Via Emilia, ivi); la figura di Giuseppe Posatini, Pinei, e la nascita della Brigata Stella Rossa (Pinei il ribelle, ivi); l'omicidio di Angelo Chiozza, operaio della Cementi Rossi (Angelo Chiozza, in «AM», aprile-maggio 1977); Emilio Canzi e il gruppo di Peli (Don Giovanni Bruschi, un prete partigiano, ivi).

29 La canzone partigiana a Piacenza, in «AM», febbraio-marzo 1976, p. 9. 
"veglia antifascista” la sera del 24 aprile, nel corso della quale i partigiani vengono invitati a raccontare e attualizzare la propria storia ${ }^{30}$, si ricomincia ad andare in montagna, con le "pedalate antifasciste" 31.

In questo nuovo modo di parlare di Resistenza, le statue di Secondo Tizzoni giocano un ruolo non secondario. Alle manifestazioni «imbalsamate», i giovani del Cam preferiscono i luoghi antiretorici, i protagonisti secondari. Grandi manifestazioni vengono organizzate al Monticello di Gazzola e a Peli di Coli, dove ai piedi delle statue si ascoltano i racconti di partigiani, come Paolo Belizzi, presidente e «fiore all'occhiello» del Cam. È proprio grazie a Belizzi che la figura di Canzi vive una nuova stagione di attenzione, assurgendo a simbolo di coerenza morale e intransigenza antifascista, contro ogni gioco di partito e compromesso di potere [Belizzi 1970; Belizzi 1983].

L'aura di Canzi è forte. Il suo personale carisma attinge alla tradizione, prevalente nel primo Novecento piacentino, del sindacalismo rivoluzionario, con la sua attitudine a tradursi in una etica comportamentale prima che in assunti teorici. Una tradizione che rimane viva nella memoria collettiva per costruire poi il cemento del primo Cln provinciale, dove Belizzi, pur essendo comunista, sceglierà di stare dalla parte di Canzi, senza seguire la linea di partito che lo voleva destituito. Belizzi riannoda il fascino esercitato da Canzi sui partigiani a quello che Angelo Faggi, seguitissimo segretario della Camera del lavoro negli anni Venti, aveva avuto sui lavoratori piacentini.

Un accostamento tra due figure di leader che non sovviene solo a Belizzi. Per salutare il ritorno alla vita politica cittadina dell'anziano Faggi nel dopoguerra, dopo anni di esilio, un cronista locale aveva significativamente usato toni che sembravano descrivere la celebre foto di Canzi:

Quando vedo Faggi per la strada [...] mi dà l'idea che sia uno arrivato chissà da dove senza un bagaglio, un pacco, senza niente, e stia per ripartirsene verso una meta qualsiasi, non importa quale, tanto per lui fa lo stesso perché quello è il suo destino: camminare [Finetti, Fontana 2005, 144].

Come scrive Gianni D’Amo, principale animatore del Comitato antifascista: «Riscoprimmo luoghi e figure della Resistenza piacentina allora trascurati, se non dimenticati» [D’Amo 2005, 17]. Lapidi e sculture diventano luoghi di pellegrinaggio, e il Comitato si dichiara «impegnato in questa strada di documentazione affinché i monumenti e i cippi che numerosi nella nostra provincia ricordano i caduti siano un vivo richiamo [alla] continuità della Resistenza» ${ }^{32}$.

30 Tacchini: la Resistenza continua, in «AM», aprile 1976, p. 5.

31 Pedalata antifascista, in «AM», giugno-luglio 1976, p. 9; In bici al monumento di Gazzola, in «Libertà», 4 giugno 1977, p. 5; Ricordare è capire dimenticare è tradire, in «AM», giugno-luglio 1977.

32 Bettola, il monumento ai partigiani trucidati, in «AM», luglio-agosto 1975, p. 7. 


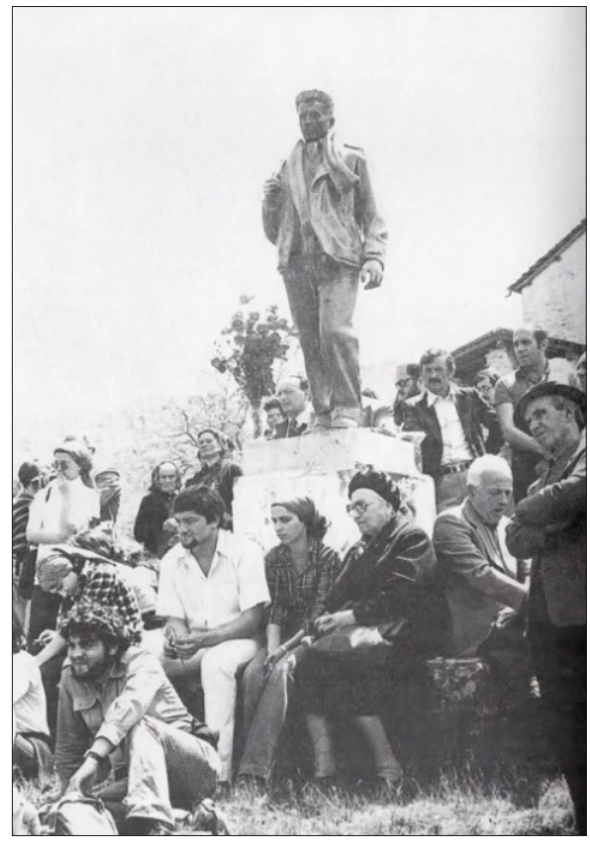

Fig. 11. Manifestazione del Cam a Peli di Coli, s.d. [Archivio Comitato antifascista militante].

Nuovo interesse per la Resistenza viene anche dall'amministrazione comunale, che nel 1975 viene riconquistata dal Partito comunista, dopo un quindicennio di giunte socialiste e democristiane. Anche a Piacenza viene così inaugurato, il 25 aprile 1976, il monumento ai caduti della Resistenza, che da almeno dieci anni le associazioni partigiane reclamavano con petizioni e lettere aperte ${ }^{33}$.

Figlia di un'epoca di forte ripensamento delle forme monumentali, la scultura realizzata da William Xerra su commissione del Consiglio federativo della Resistenza è pensata come «un'opera che non è monumento ma ne va ben oltre» ${ }^{34}$. Il dolmen, inserito in un'aiuola, si compone di un trilite in marmo e intende rappresentare un «monumento funerario universale [...], che esula da ogni rappresentazione retorica e tradizionale, si inserisce con autenticità nella cultura del nostro tempo per la sua struttura plastica e concettuale, è aperta all'aria, al vento e alla fantasia» [Carrà 1976, 3-7].

I toni utilizzati riecheggiano la polemica, nodale per il rapporto tra monumenti e Resistenza, sul monumento nazionale di Cuneo. Là un bando indetto dall'amministrazione comunale aveva fatto sorgere, a partire dal 1963, un vivace dibattito circa l'opportunità di monumentalizzare la Resistenza. Su riviste specialistiche e periodici nazionali, artisti e intellettuali si erano divisi tra i sostenitori della necessità di celebrare artisticamente la lotta partigiana e coloro che, al contrario, ritenevano che una eccessiva monumentalizzazione contribuisse a dissolvere, in una retorica con-

\footnotetext{
33 ANPIPC, b. 112, Erezione cippi e monumenti, fasc. Monumento al partigiano.

34 Ettore Carrà, s.d., in ANPIPC, b. 112, Erezione cippi e monumenti, fasc. Monumento ai caduti della Resistenza.
} 
Fig. 12. Statua di Emilio Canzi a Peli di Coli [foto Lucia Baldini].

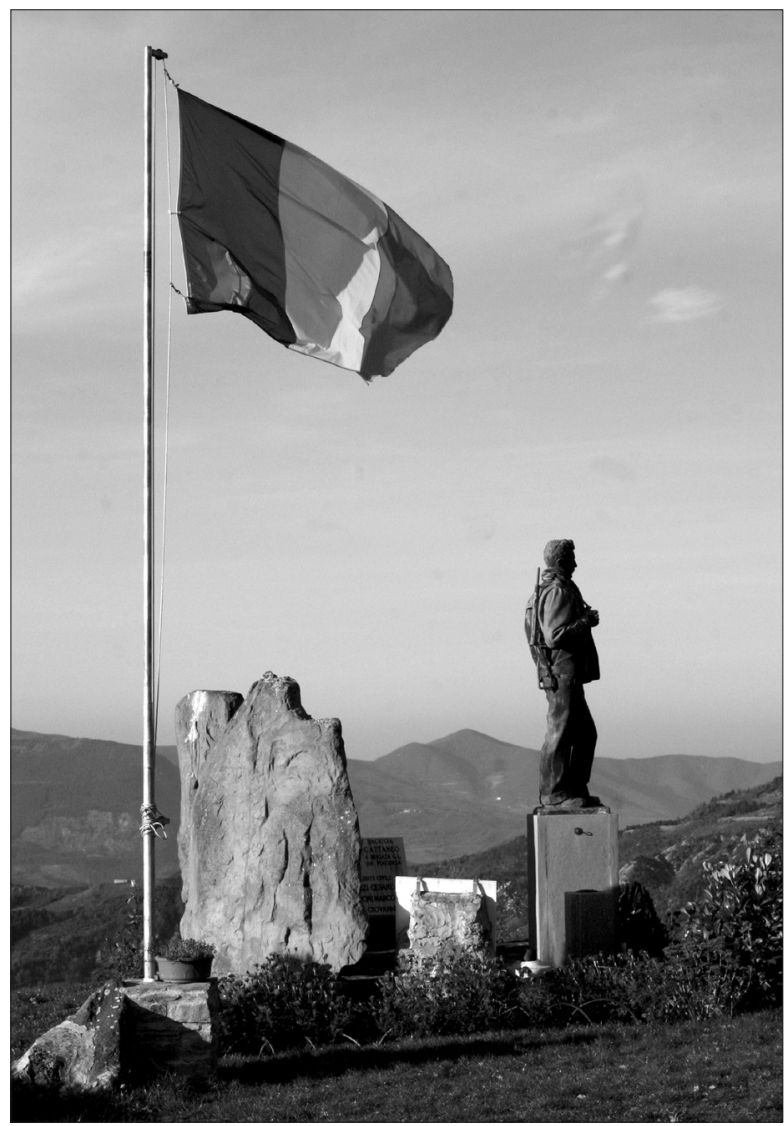

venzionale e stereotipata, il patrimonio ideale e lo slancio vitale della Resistenza. Con questo problema aperto si misurano artisti che vengono incaricati di realizzare monumenti di questo tipo: le loro scelte fanno spesso riferimento a «non monumenti», «luoghi di memoria», «percorsi sacri», «convivenza di scultura e natura», «santuari dell'antichità» [Finocchi 1995, 25-27; Finocchi 1999, 76-81]. Il dolmen di Xerra non manca comunque di suscitare polemiche in città. C'è chi avrebbe preferito un'altra collocazione ${ }^{35}$, chi pensa che sia eccessivamente concettuale e che rischi di non essere compreso dalla gente comune ${ }^{36}$, chi - come i giovani del Cam - avrebbe preferito la figura di un partigiano armato, come quello realista e didascalico realizzato a Parma da Marino Mazzacurati ${ }^{37}$. A difendere il valore dell'opera è Ludovico Muratori, che presiede il comitato preposto alla costruzione, che ritiene sia ora di ovviare alla mancanza nella città di Piacenza di un monumento ai partigiani caduti, presente da anni nelle vicine Parma (col già citato monumento di Mazzacurati inaugurato nel 1956) e Reggio Emilia (col monumento di Remo Brio-

35 Ivi, Lettera di G. Valentini a Felice Trabacchi, 13 marzo 1976.

36 Ivi, Lettera di Andrea Cammarosano, Camma, a Felice Trabacchi, s.d.

37 La Resistenza del fucile e quella del dolmen, in «AM», aprile, 1976, p. 4. 
schi inaugurato nel 1958). Un anno prima Muro aveva progettato in prima persona un monumento, dedicato ai caduti dell'eccidio di Rio Farnese e posto a Bettola, nel luogo della strage. Il comandante partigiano aveva scelto una rappresentazione astratta, dove dei blocchi di calcestruzzo disposti a scacchiera creavano 21 spazi vuoti, come 21 erano le vite dei compagni lì fucilati.

\section{Conclusioni}

Nel secondo dopoguerra, la scultura monumentale non ha, per diffusione o rilevanza nella vita pubblica, il peso che aveva avuto dopo il primo conflitto. Le statue - che servono a ricordare eccidi e massacri, onorare il sacrificio dei caduti o celebrare il ruolo dei partigiani - sono sparse sul territorio nazionale in maniera non uniforme. Nella persistente difficoltà di trovare anche per la Resistenza forme di commemorazione nazionale, le statue partigiane sono spesso patrimoni locali, che raccontano storie di provincia, sorte grazie a sottoscrizioni popolari e all'insistenza di sezioni comunali delle associazioni partigiane.

Di queste spinte, che dal dopoguerra mutano e si trasformano, il caso piacentino è un buon esempio. I documenti e la stampa locali restituiscono l'impegno delle associazioni del territorio per costruire i monumenti e vivificarli attraverso pratiche commemorative e ritrovi periodici. Qui, il ricordo della Resistenza trova un sensibile interprete in Secondo Tizzoni, che con la sua scelta "insolita", consegna alla città opere di grande efficacia comunicativa, capaci di parlare della storia della Resistenza attraverso le generazioni. Il suo lavoro - ingiustamente confinato all'ambito provinciale - mette a fuoco alcune particolarità artistiche e storiche, e ci mostra l'importanza della fotografia nella costruzione della memoria della lotta partigiana. Le serie fotografiche, spesso scattate all'indomani della Liberazione o dopo la smobilitazione, ritraggono i partigiani in armi, vittoriosi, esattamente come volevano essere ricordati, e fissano immagini iconiche della Resistenza che contribuisce a plasmare memorie e sculture [Mignemi 1995]. Numerose serie fotografiche e fotomontaggi realizzati nel dopoguerra diventano oggetto di mostre ed esposizioni, capaci di mostrare al paese e al mondo l'impegno dei partigiani e correggere l'immagine di un'Italia fortemente compromessa con il fascismo e con le guerre di aggressione [Mignemi, Solaro 2005].

Anche le statue dei partigiani piacentini, come i cavalli del Mochi, sono ormai un importante simbolo identitario locale.

Mentre, a partire dagli anni Ottanta il "paradigma antifascista” viene pesantemente attaccato [Focardi 2005, 56-93] e la "religione civile" della Resistenza viene rimessa in discussione anche nei suoi simboli e nelle sue traduzioni artistiche [Gundle 2000], nascono nuove forme di mobilitazione basate sulla storia partigiana.

Dopo diverse denunce sulla «emarginazione dei partigiani», in vista del sessantesimo anniversario della Liberazione, Ludovico Muratori, Muro, decide di rivolgersi agli organi di stampa locale e chiama a raccolta tutti i giovani, «gli under 30», invitandoli a farsi carico di documenti, memorie, commemorazioni. Non 

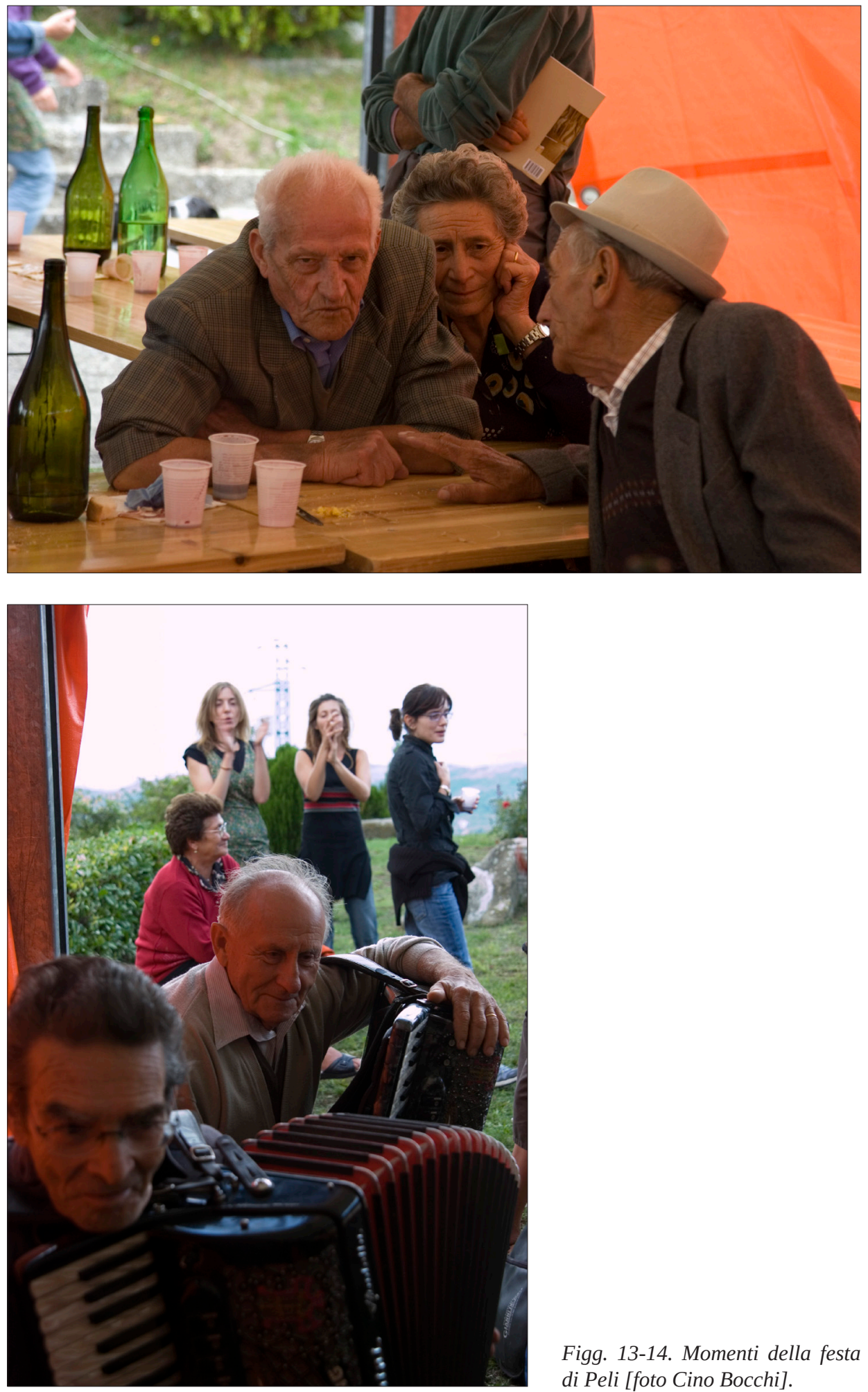

Figg. 13-14. Momenti della festa di Peli [foto Cino Bocchi]. 
sono in pochi a rispondere a quell'appello ${ }^{38}$. Pochi giorni dopo Muratori muore, e sorge il Comitato giovani Anpi comandante Muro, tra i cui obiettivi c'è proprio il rilancio della storia della Resistenza locale.

Se per la baby boom generation del Cam il dialogo con la Resistenza è fitto e continuo, la generazione nata negli anni Ottanta percepisce un rapporto più labile e precario con il passato, e sente la necessità di riappropriarsi attivamente della storia [Pivato 2007, VIII]. Insieme a piazza Cavalli, luogo di un tradizionale concerto che - sulla scia di quelli organizzati a Casa Cervi - è divenuto uno dei simboli della "festa del 25 aprile", gli altri luoghi scelti dal neonato Comitato sono proprio le statue di Tizzoni.

Nuovo slancio è stato dato alla commemorazione della battaglia del Monticello, e una festa partigiana è stata a lungo organizzata ai piedi della statua di Canzi a Peli. Il monumento ormai non si trova più nella posizione originale. Nel 1983 la statua è stata rimossa dal basamento, ormai consunto, e posta in un complesso monumentale più ampio, composto con grandi sassi e realizzato dallo scultore Paolo Perotti su commissione dell'amministrazione comunale di Coli. Una cornice sicuramente più "importante” ma che toglie alla scultura originaria l'apertura allo spazio, lo sguardo prospettico, la vocazione antiretorica. Lo spostamento impedisce oggi di leggere nel loro insieme le componenti comunicative del complesso monumentale. In particolare non è stato possibile analizzare il basamento ed eventuali epigrafi originali, che avrebbero potuto gettare luce sugli intenti e le motivazioni di committenti e realizzatori.

Eppure Canzi continua a camminare.

Le commemorazioni organizzate a Peli a partire dal 2005, con musica, canti, pranzi al sacco, interviste ai partigiani, spettacoli teatrali, trekking, insistono soprattutto sul - in realtà non sempre lineare - ruolo della gente della montagna nel sostenere la Resistenza; e sulla figura di Emilio Canzi come emblema dell'unitarietà della Resistenza piacentina.

Mentre i luoghi della città fanno parte della quotidianità e spesso vengono dati per scontati, l'atto di salire in montagna, viene sentito come una sorta di "pellegrinaggio laico”, capace di immergere non solo nella storia ma anche in parte nell'esperienza umana dei partigiani ${ }^{39}$.

38 Così emarginano gli ex partigiani, in «Libertà», 2 novembre 2004, p. 8.

39 Questo saggio è stato scritto durante la pandemia, con accesso limitato a archivi e biblioteche. La mia più profonda riconoscenza a coloro che mi hanno inviato fotografie, documenti, suggerimenti: Sandro Agnelli, Francesca Agosti, Gianni Agosti, Francesco Barilli, Paola Boccalatte, Cino Bocchi, Piero Bonvini e Gruppo ricerca immagine di Bettola, Mirco Carrattieri, Maurizio Cavalloni, Fiamma Chessa, Fausto Cossu, Gianni D’Amo, Gabriele Dadati, Eugenio Ermeti, Claudio Mazzolani, Tomaso Montanari, Rosella Tiadina, Paolo Tizzoni, Roberto Tonelli, Alessandro Pigazzini, Romano Repetti, Claudio Silingardi. 


\section{Bibliografia}

Agosti, Vescovi 2021

Giovanni Agosti, Alberta Vescovi, E verrà l'alba... Il Valoroso: una vita partigiana, Piacenza, Vicolo del Pavone, 2021.

Baioni 2020

Massimo Baioni, Vedere per credere. Il racconto museale dell'Italia unita, Roma, Viella, 2020.

Baldissara, Pezzino 2009

Luca Baldissara, Paolo Pezzino, Il massacro. Guerra ai civili a Monte Sole, Bologna, il Mulino, 2009.

Belizzi 1970

Paolo Belizzi, Il gerarchetto, Piacenza, Gallarati, 1970.

Belizzi 1983

Paolo Belizzi, Quelle che non fanno storia: pagine della cospirazione antifascista a Piacenza, Piacenza, Nazionale, 1983; II ediz. a cura di Gianni D’Amo, TortonaPiacenza, Vicolo del Pavone, 2005.

Bianchera s.d.

Dario Bianchera, Censimento monumenti, cippi ed altre opere dedicate ai partigiani caduti nella Resistenza piacentina, Piacenza, ANPI, s.d.

Bianchini, Lolli 1997

Letteratura e Resistenza, a cura di Andrea Bianchini, Francesca Lolli, Bologna, Clueb, 1997.

Bonvicini 2002

Gyonata Bonvicini, La fotografia, in Storia di Piacenza, vol. 6, tomo I, Piacenza, Tipleco, 2002, pp. 585-606.

Bracco 2015

Milano nella Grande guerra. La memoria dei caduti e il Cimitero Monumentale, a cura di Barbara Bracco, Milano, Bilblion, 2015.

Carrà 1976

Ettore Carrà, Ai caduti della Resistenza: Piacenza 25 aprile 1976, Piacenza, Consiglio provinciale federativo della Resistenza, 1976.

Cavalloni, Panciroli 2016

Maurizio Cavalloni, Daniele Panciroli, Gianni Croce. Fotografie 1921-1964, Piacenza, Museo per la fotografia e la comunicazione visiva, 2016.

Cigognetti, Servetti, Sorlin 1996

L'immagine della resistenza in Europa 1945-1960. Letteratura, cinema, arti figurative, a cura di Luisa Cigognetti, Lorenza Servetti, Pierre Sorlin, Bologna, Il Nove, 1996.

Coles, Weinberg 1964

Civil Affairs. Soldiers Become Governors, a cura di Harry L. Coles e Albert K. Weinberg, Washington, Office of the Chief of Military History, 1964.

\section{Concarotti 1984}

Ennio Concarotti, Piacenza 40-45: il dramma di una città. Come vissero e sopravvissero i piacentini negli anni della guerra, della caduta del fascismo, dell'occupazione tedesca, della lotta civile e dell'avvento della democrazia, Piacenza, Humanitas, 1984.

\section{Contini 1997}

Giovanni Contini, La memoria divisa, Milano, Rizzoli, 1997. 


\section{Cooke 2015}

Philip Cooke, L'eredità della Resistenza. Storia, cultura, politiche dal dopoguerra a oggi, Roma, Viella, 2015.

Crainz 2000

Guido Crainz, I programmi televisivi sul fascismo e la Resistenza, in Fascismo e antifascismo. Rimozioni, revisioni, negazioni, a cura di Enzo Collotti, Roma-Bari, Laterza, 2000, pp. 463-492.

D’Amelia 2005

Marina D’Amelia, La mamma, Bologna, il Mulino, 2005.

D’Amo 2005

Gianni D’Amo, La coerenza difficile, prefazione alla II ediz. di Paolo Belizzi, Quelle che non fanno storia: pagine della cospirazione antifascista a Piacenza, a cura di Gianni D’Amo, Tortona-Piacenza, Vicolo del Pavone, 2005.

De Luna 1995

Giovanni De Luna, Le identità, in Fascismo/antifascismo. Le idee, le identità, a cura di Giovanni De Luna, Marco Revelli, Firenze, La Nuova Italia, 1995, pp. 141-148.

Dogliani 1995

Patrizia Dogliani, Monumenti alla Resistenza. Bologna e il suo territorio, in La premiata Resistenza. Concorsi d'arte nel dopoguerra in Emilia-Romagna, Bologna, Grafis, 1995, pp. 21-38.

Dogliani 2006

Patrizia Dogliani, I monumenti e le lapidi come fonti, in Storia d'Italia nel secolo ventesimo. Strumenti e fonti, a cura di Claudio Pavone, vol. I, Roma, Archivi di Stato, 2006, pp. 261-275.

Dondi 2004

Mirco Dondi, La Resistenza tra unità e conflitto. Vicende parallele tra dimensione nazionale e realtà piacentina, Milano, Bruno Mondadori, 2004.

Falaschi 1976

Giovanni Falaschi, La Resistenza armata nella narrativa italiana, Torino, Einaudi, 1976.

Filippetta 2018

Giuseppe Filippetta, L'estate che imparammo a sparare. Storia partigiana della Costituzione, Milano, Feltrinelli, 2018.

Finocchi 1995

Anna Finocchi, L'atto disperato e costante della ricerca, in Mario Negri. Catalogo delle sculture, a cura di Anna Finocchi, Bergamo, Bolis, 1995, pp. 7-12.

Finocchi 1999

Anna Finocchi, I monumenti: luogo e forma della memoria, in La Resistenza tra storia e memoria, a cura di Nicola Gallerano, Milano, Mursia, 1999, pp. 72-83.

Focardi 2005

Filippo Focardi, La guerra della memoria. La Resistenza nel dibattito politico italiano dal 1945 a oggi, Roma-Bari, Laterza, 2005.

Focardi 2020

Filippo Focardi, Nel cantiere della memoria. Fascismo, Resistenza, Shoah, Foibe, Roma, Viella, 2020.

Finetti, Fontana 2005

Claudia Finetti, Severina Fontana, Le ragioni del lavoro. Studi per la storia della Camera del lavoro di Piacenza, Piacenza, Tipolito Farnese, 2005.

Forgacs 1999

David Forgacs, Fascism and Anti-Fascism Reviewed: Generations, History and Film 
in Italy after 1968, in European Memories of World War II, a cura di Helmut Peitsch, Charles Berdett, Claire Gorrara, New York-Oxford, Berghahn, 1999, pp. 185-199.

Forlani 1993

Alessandro Forlani, Francesco Daveri. Un cristiano per la libertà, Piacenza, Emilstampa, 1993.

Franzolini 2006

Monumenti ai caduti in guerra nella provincia di Novara e nella provincia del Verbano Cusio Ossola, a cura di Sabino Franzolini, Novara, Ass. Naz. Combattenti e Reduci, 2006.

Fugazza 2000

Stefano Fugazza, Secondo Tizzoni: disegni e sculture, Piacenza, Tipleco, 2000.

Galmozzi 1986

Luciano Galmozzi, Monumenti alla libertà. Antifascismo, resistenza e pace nei monumenti italiani dal 1945 al 1985, Milano, La Pietra, 1986.

Gambetta 2000

William Gambetta, “Almirante non parlerà”. Radici e caratteri dell'antifascismo militante parmense, in Parma dentro la rivolta. Tradizione e radicalità nelle lotte sociali e politiche di una città dell'Emilia rossa 1968-1969, Milano, Punto Rosso, 2000, pp. 277-330.

Giovannini Luca, Tabor 2017

Una memoria per immagini: guerra e Resistenza nelle fotografie di Ettore Serafini, a cura di Alessandra Giovannini Luca, Davide Tabor, Milano, FrancoAngeli, 2017.

Gundle 2000

Gundle Stephen, The 'Civic Religion' of the Resistance in Post-War Italy, in «Modern Italy», 5, 2 (2000), pp. 113-132.

Hullis 2014

Shaun Hullis, Captains Courageous. Donny Mackenzie, Gunner Gregg \& the Liberation of the Nure Valley, Great Britain, CreateSpace Independent Publishing Platform, 2014.

Isnenghi 2005

Mario Isnenghi, Le guerre degli italiani. Parole, immagini, ricordi 1848-1945, Bologna, il Mulino, 2005 (I ed. 1989).

Labanca 2010

Nicola Labanca, Pietre di guerra. Ricerche su monumenti e lapidi in memoria del primo conflitto mondiale, Milano, Unicopli, 2010.

MacKenzie 2000

William J. M. MacKenzie, The Secret History of SOE, 1940-1945, London, St. Ermin's Press, 2000.

Matteo 2020

Jessica Matteo, Parole pubbliche e memorie private - L'antifascismo militante a Roma negli anni Settanta, Roma, Polis SA, 2020.

Meloni, Menzani 2019

Iara Meloni, Giovanni Battista Menzani, Ribelli all'ombra della Pietra, Piacenza, Officine Gutenberg, 2019.

Meloni 2019

Iara Meloni, L'altra giustizia. La Corte di assise straordinaria di Piacenza (19451947), Piacenza, Le Piccole Pagine, 2019.

Mignemi 1995

Adolfo Mignemi, Storia fotografica della Resistenza, Torino, Bollati Boringhieri, 1995. 
Mignemi, Solaro 2005

Adolfo Mignemi, Gabriella Solaro, Un'immagine dell'Italia. Resistenza e ricostruzione. Le mostre del dopoguerra in Europa, Milano, Skira, 2005.

Montanari 2020

Tomaso Montanari, Capolavori fuori dal centro. I Cavalli di Piacenza di Francesco Mochi, Milano, Skira, 2020.

Morsia 2001

Daniela Morsia, Cinquant'anni di storiografia della Resistenza piacentina, in La cultura della Resistenza: storiografia e identità civile in Emilia-Romagna, a cura di Brunella Dalla Casa, Alberto Preti, Bologna, Il Nove, 2001, pp. 191-201.

Mosse 1990

George L. Mosse, Le guerre mondiali. Dalla tragedia al mito dei caduti, Roma-Bari, Laterza, 1990.

Oltremonti 2012

Claudio Oltremonti, Nelle SPIRE del Regime. Upi, Questura, OVRA, MGIR, Missioni Alleate, Intelligence partigiana a Piacenza (1943-1945), s.n., 2012.

Paggi 1999

Leonardo Paggi, Le memorie della Repubblica, Firenze, La Nuova Italia, 1999.

Paggi 2009

Leonardo Paggi, Il popolo dei morti. La Repubblica italiana nata dalla Resistenza, Bologna, il Mulino, 2009.

Panni 1978

Giuseppe Panni, La $61^{a}$ Brigata autonoma d'assalto Mazzini e la Brigata Pietro Inzani in Val Nure e in Val d'Arda, Piacenza, TEP-Gallarati, 1978.

Piffer 2010

Tommaso Piffer, Gli Alleati e la Resistenza italiana, Bologna, il Mulino, 2010.

Pivato 2005

Stefano Pivato, Bella ciao. Canto e politica nella storia d'Italia, Roma-Bari, Laterza, 2005.

Pivato 2007

Stefano Pivato, Vuoti di memoria. Usi e abusi della storia nella vita pubblica italiana, Roma-Bari, Laterza, 2007.

Poli 1990

Francesco Poli, La smonumentalizzazione della scultura, in Il lauro e il bronzo. La scultura celebrativa in Italia 1800-1900, a cura di Maurizio Corgnati, Gianlorenzo Mellini, Francesco Poli, Catalogo della mostra, Torino, s.n., 1990, pp. 23-29.

Portelli 1999

Alessandro Portelli, L'ordine è già stato eseguito. Roma, le Fosse Ardeatine, la memoria, Roma, Donzelli, 1999.

Ridolfi 2003

Maurizio Ridolfi, Le feste nazionali, Bologna, il Mulino, 2003.

Schwarz 2010

Guri Schwarz, Tu mi devi seppellir. Riti funebri e culto nazionale alle origini della Repubblica, Torino, Utet, 2010.

Silingardi 1987

Claudio Silingardi, Emilio Canzi e Savino Fornasari dall'emigrazione libertaria in Francia alla rivoluzione spagnola, in «Studi Piacentini», 1 (1987), pp. 11-38. 
Silingardi 1991

Claudio Silingardi, Emilio Canzi e la crisi del Comando unico piacentino (19441945), in «Studi Piacentini», 10 (1991), pp. 7-48.

Silingardi 2003

Claudio Silingardi, Emilio Canzi, in Dizionario biografico degli anarchici italiani, Pisa, BFS, 2003, https://www.bfscollezionidigitali.org/entita/13422-canzi-emilio.

Sprega 1998

Franco Sprega, Il filo della memoria. Fatti e cronache di Fiorenzuola dal movimento socialista agli albori della Resistenza, Piacenza, Tipleco, 1998.

Testimonianze incise 1999

Testimonianze incise nel marmo. Monumenti, cippi e lapidi ai caduti della Resistenza piacentina, Piacenza, ANPI, 1999.

Vidotto, Tobia, Brice 1998

La memoria perduta: i monumenti ai caduti della Grande guerra a Roma e nel Lazio, a cura di Vittorio Vidotto, Bruno Tobia, Catherine Brice, Roma, Argos, 1998.

Wardi 1993

Dina Wardi, Le “candele” della memoria. I figli dei sopravvissuti all'Olocausto: traumi, angosce, terapia, Milano, Sansoni 1993 (ed. or. 1992).

Winter 2014

Jay Winter, Il lutto e la memoria. La grande guerra nella cultura europea, Bologna, il Mulino, 2014 (ed. or. 1999).

Winterhalter 2010

Cecilia Winterhalter, Raccontare e inventare: storia, memoria e trasmissione storica delle Resistenza armata in Italia, Bern, Lang, 2010. 